\title{
ARTICLE
}

\section{Cycloastragenol upregulates SIRT1 expression, attenuates apoptosis and suppresses neuroinflammation after brain ischemia}

\author{
Man $\mathrm{Li}^{1,2}$, Shi-chun $\mathrm{Li}^{1,2}$, Bao-kai Dou ${ }^{1,2}$, Ying-xiang Zou ${ }^{1,2}$, Hao-zhen Han ${ }^{3,4}$, Dong-xiang Liu ${ }^{3,4}$, Zun-ji Ke ${ }^{2}$ and Zhi-fei Wang ${ }^{1,2}$
}

\begin{abstract}
Cycloastragenol (CAG) is the active form of astragaloside IV isolated from Astragalus Radix, which displays multiple pharmacological effects. Silent information regulator 1 (SIRT1), a class III histone deacetylase, has been shown to play an important role in neuroprotection against cerebral ischemia. In this study, we investigated whether CAG protected against ischemic brain injury and, if so, whether the beneficial effects were associated with the regulation of SIRT1 in the ischemic brain. Mice were subjected to $45 \mathrm{~min}$ of middle cerebral artery occlusion (MCAO) followed by reperfusion. CAG $(5,10,20 \mathrm{mg} / \mathrm{kg})$ was injected intraperitoneally at the onset of reperfusion, $12 \mathrm{~h}$ later and then twice daily for up to three days. CAG dose-dependently reduced brain infarct volume, significantly ameliorated functional deficits, and prevented neuronal cell loss in MCAO mice. Meanwhile, CAG significantly reduced matrix metalloproteinase- 9 activity, prevented tight junction degradation and subsequently ameliorated blood-brain barrier disruption. Moreover, CAG significantly upregulated SIRT1 expression in the ischemic brain but did not directly activate its enzymatic activity. Concomitant with SIRT1 upregulation, CAG reduced p53 acetylation and the ratio of Bax to Bcl-2 in the ischemic brain. CAG also inhibited NF-kB p65 nuclear translocation. As a result, CAG suppressed the mRNA expression of pro-inflammatory cytokines, including TNF- $\alpha$ and IL-1 $\beta$, and inhibited the activation of microglia and astrocytes in the ischemic brain. Our findings suggest that CAG is neuroprotective against ischemic brain injury in mice and that its beneficial effect may involve SIRT1 upregulation and the inhibition of apoptosis and neuroinflammation in the ischemic brain.
\end{abstract}

Keywords: cycloastragenol; SIRT1; apoptosis; neuroinflammation; cerebral ischemia

Acta Pharmacologica Sinica (2020) 41:1025-1032; https://doi.org/10.1038/s41401-020-0386-6

\section{INTRODUCTION}

Stroke is one of the leading causes of death and adult disability worldwide. Ischemic stroke is the most common type of stroke and accounts for more than $70 \%$ of all strokes in China [1]. To date, recombinant tissue plasminogen activator (rtPA) is the only clinically approved treatment for acute ischemic stroke. However, its narrow therapeutic window and safety concerns limit the clinical use of rtPA. Therefore, it is crucial to develop new therapies for ischemic stroke.

Silent information regulator 1 (SIRT1) is a class III histone deacetylase, and its enzymatic activity depends on oxidized nicotinamide adenine dinucleotide. SIRT1 is expressed throughout the body and deacetylates histones and nonhistone proteins to regulate cell metabolism in response to stress [2]. The neuroprotective properties of SIRT1 have been demonstrated in many neurodegenerative disorders, including neurodegenerative and cerebrovascular diseases [3-5].

Sirt1 knockout mice exhibit larger brain infarction than wild-type littermates following middle cerebral artery occlusion (MCAO) [6]. In contrast, Sirt1 overexpression attenuates hippocampal damage in mice subjected to cerebral ischemia [7]. On the other hand, resveratrol, a natural SIRT1 activator, has been shown to exert neuroprotective effects via SIRT1 pathways in in vitro and in vivo models of cerebral ischemia, while the inhibition of SIRT1 activation by sirtinol can abolish the neuroprotective effects of resveratrol $[8,9]$. Together, these findings suggest that SIRT1 has emerged as a promising target for neuroprotection against cerebral ischemia $[4,5]$.

Astragalus radix is a traditional Chinese herbal medicine that is commonly used to treat cardiovascular diseases, diabetes and cancers in China [10]. Astragaloside IV is the major active component of Astragalus radix and is responsible for most of the observed effects of this herbal medicine. Most astragaloside IV is metabolized to cycloastragenol (CAG, Fig. 1a) in vivo [11]. Therefore, CAG is regarded as an active form of astragaloside IV [12]. CAG has been demonstrated to possess multiple pharmacological effects, including telomerase-activating, anti-aging, antiapoptotic, and anti-inflammatory effects [12-14]. The effect of CAG against cerebral ischemia remains elusive. The present study investigated whether CAG can protect against ischemic brain injury and, if so, whether the beneficial effect is associated with the upregulation of SIRT1 in the ischemic brain.

\footnotetext{
${ }^{1}$ School of Basic Medical Sciences, Shanghai University of Traditional Chinese Medicine, Shanghai 201203 , China; ${ }^{2}$ Academy of Integrative Medicine, Shanghai University of Traditional Chinese Medicine, Shanghai 201203, China; ${ }^{3}$ Department of Pharmacology III, Shanghai Institute of Materia Medica, Chinese Academy of Sciences, Shanghai 201203, China and ${ }^{4}$ University of Chinese Academy of Sciences, Beijing 100049, China

Correspondence: Zhi-fei Wang (zfwang@shutcm.edu.cn)

These authors contributed equally: Man Li and Shi-chun Li
}

Received: 9 November 2019 Accepted: 19 February 2020

Published online: 16 March 2020 


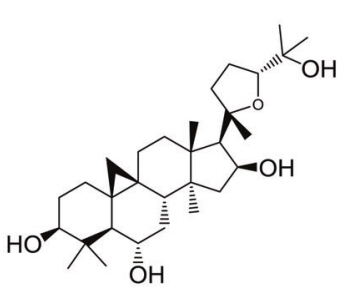

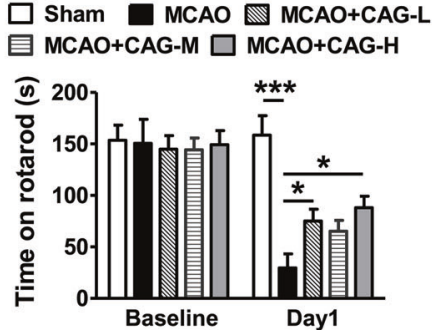

d

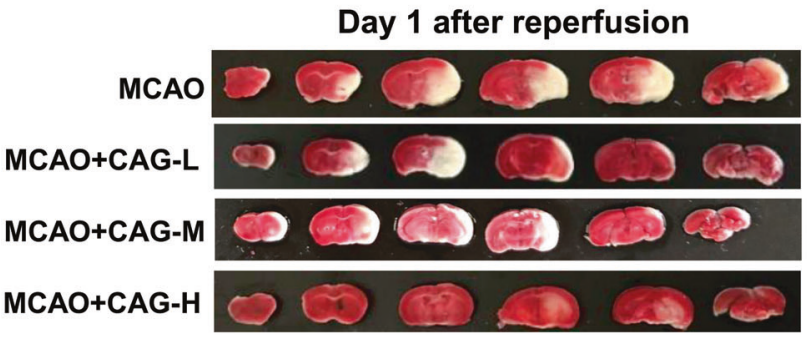

C

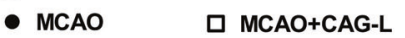

$\triangle$ MCAO+CAG-M O MCAO+CAG-H

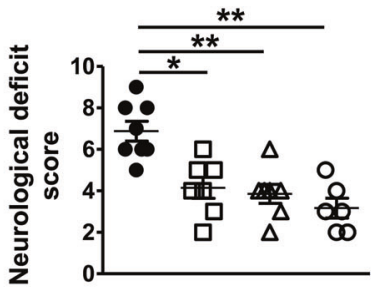

e

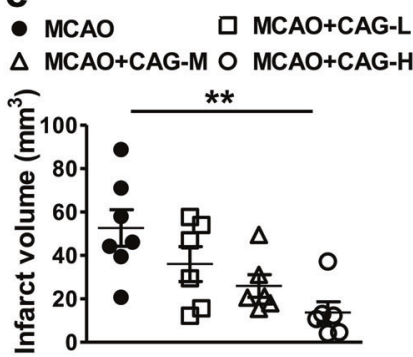

Fig. 1 CAG attenuated functional deficits and reduced the brain infarct volume in MCAO mice on Day 1 after reperfusion. a The chemical structure of CAG. b Compared with vehicle-treated MCAO mice, MCAO mice treated with CAG (5 or $20 \mathrm{mg} / \mathrm{kg}) \mathrm{spent}$ a much longer time on the accelerating rotarod on Day 1. c CAG reduced neurological deficit scores in a dose-dependent manner. $\mathbf{d}$, e TTC staining showed that $20 \mathrm{mg} / \mathrm{kg}$ CAG significantly reduced the brain infarct volume in MCAO mice on Day 1. CAG-L: $5 \mathrm{mg} / \mathrm{kg}, \mathrm{CAG}-\mathrm{M}: 10 \mathrm{mg} / \mathrm{kg}, \mathrm{CAG}-\mathrm{H}: 20 \mathrm{mg} / \mathrm{kg}$. ${ }^{*} P<0.05 ;{ }^{* *} P<0.01 ;{ }^{* *} P<0.001 ; n=6-8$ per group

\section{MATERIALS AND METHODS}

\section{MCAO and drug administration}

All animal protocols and procedures were approved by the Experimental Animal Ethical Committee, Shanghai University of Traditional Chinese Medicine. Male C57BL/6 mice (23-26 g, Vital River Laboratory Animal Technology Co., Ltd., Beijing, China) underwent right MCAO under inhalational anesthesia $(1.5 \%$ halothane) as previously described [15, 16]. After $45 \mathrm{~min}$, the silicon-coated suture was withdrawn to initiate reperfusion. CAG (purity $>98 \%$, Yuanye Biotechnology, Shanghai, China) was dissolved in DMSO and then diluted with $2 \%$ Tween 20 in PBS. The final concentration of DMSO was $5 \%$. The animals were randomly divided into the sham, MCAO, and MCAO + CAGtreated groups. CAG $(5,10$ or $20 \mathrm{mg} / \mathrm{kg}$, i.p.) was injected immediately after reperfusion, $12 \mathrm{~h}$ later, and then twice daily for up to three days.

\section{Behavioral tests}

All behavioral tests were performed by investigators blinded to the treatment conditions. The accelerating rotarod test was performed and neurological deficit scores were assessed $24 \mathrm{~h}$ and $72 \mathrm{~h}$ after reperfusion, and gait analysis was performed $72 \mathrm{~h}$ after reperfusion to evaluate the effects of CAG on the functional deficits of MCAO mice. The accelerating rotarod test and neurological deficit score test were performed as previously described [16].

Gait analysis was performed with the CatWalk 10.5 Analysis System (Noldus Information Technology, Wageningen, Netherlands). Constantly changing paw images were captured by a highspeed camera underneath a glass plate when mice walked across the plate. Each mouse was required to walk across the plate at least three times without pausing. Mice that were unable to complete the test due to serious injury and deficits were excluded. A series of gait parameters were calculated automatically when the system identified and marked each footprint made.

Assessment of infarct volume

Infarct volume was evaluated by $\Pi \mathrm{TC}$ and Nissl staining $24 \mathrm{~h}$ and $72 \mathrm{~h}$ after reperfusion, respectively. For TTC staining, the brain was cut into six coronal sections $(1 \mathrm{~mm})$, stained with $2 \% \pi \mathrm{TC}$ at $37^{\circ} \mathrm{C}$, and fixed in $10 \%$ formaldehyde. For Nissl staining, frozen brain sections $(30 \mu \mathrm{m})$ were dried overnight and then immersed in $0.1 \%$ cresyl violet at $37{ }^{\circ} \mathrm{C}$ for $10 \mathrm{~min}$. After rinsing with distilled water, the sections were dehydrated in $95 \%$ ethanol, submerged in xylene, and then mounted with neutral balsam. Images were captured using the Precipoint M8 digital scanning microimaging system (Precipoint, Germany) and analyzed using ImageJ (free download at http://rsbweb.nih.gov/ij/).

\section{Immunohistochemistry}

Free-floating sections $(30 \mu \mathrm{m})$ were incubated with rabbit antiNeuN (1:1000, Abcam, Cambridge, MA, USA), rabbit anti-GFAP (1:1000, Abcam) or rabbit anti-lba1 (1:1000, Wako, Chuo-ku, Osaka, Japan) followed by biotinylated anti-rabbit IgG (Yeasen, Shanghai, China) and then streptavidin-biotin peroxidase complex (Yeasen). Images were captured using the Precipoint M8 digital scanning microimaging system.

Evans blue extravasation

Evans blue (Sigma-Aldrich, St. Louis, MO, USA) extravasation was used to evaluate blood-brain barrier (BBB) permeability as previously described [17]. The brain was cut into six coronal sections $(1 \mathrm{~mm})$ to evaluate the spatial distribution of Evans blue leakage.

Gelatin zymography

The activities of matrix metalloproteinase (MMP)-2 and MMP-9 were measured by gelatin zymography as described previously [17].

RNA extraction and real-time PCR

RNA extraction and real-time PCR were performed as previously described [17]. The primer sequences for Sirt1 were $5^{\prime}$-GAATGGTCT TGGGTCTIT-3' (forward) and 5'-GGGTTTCTGTCTCCTGTG-3' (reverse). The primer sequences for TNF-a were $5^{\prime}$-CTTGTTGCCTCCTCT TाTGCTTA-3' (forward) and 5'-CTTATTCTCTCAATGACCCGTAG-3' (reverse). The primer sequences for IL-6 were $5^{\prime}$-TCACAGAAGGAG TGGCTAAGGACC- $3^{\prime}$ (forward) and 5'-ACGCACTAGGTTTGCCGAGTAG AT- $3^{\prime}$ (reverse). The primer sequences for IL-1 $\beta$ were $5^{\prime}$-ATTG 
TGGCTGTGGAGAAG-3' (forward) and 5'-AAGATGAAGGAAAAGAAG GTG-3' (reverse). The primer sequences for $\beta$-actin were $5^{\prime}$-TGTCC ACCTTCCAGCAGAT-3' (forward) and 5'-CTCAGTAACAGTCCGCCTA GA-3' (reverse). The mRNA levels were normalized against $\beta$-actin and presented as $2^{-\Delta \Delta C T}$.

Western blotting

The protein levels of Occludin, ZO-1, SIRT1, Bax, Bcl-2, NF-kB p65, histone $\mathrm{H} 3$ and $\beta$-actin were detected by Western blotting. For p65, nuclear extracts were prepared using a Nuclear and
Cytoplasmic Protein Extraction Kit (Beyotime, Shanghai, China) according to the manufacturer's protocol. The primary antibodies were as follows: rabbit anti-Occludin (1:2000, Proteintech Group, Chicago, IL, USA), rabbit anti-ZO-1 (1:500, Thermo Fisher Scientific, Waltham, MA, USA), rabbit anti-Sirt1 (1:3000, Millipore, Billerica, MA, USA), mouse anti-Bax (1:500, Santa Cruz Biotechnology, Santa Cruz, CA, USA), rabbit anti-Bcl-2 (1:1000, Santa Cruz Biotechnology), mouse anti-p65 (1:1000, Cell Signaling Technology, Boston, MA, USA), rabbit anti-histone H3 (1:1000, Abcam), and mouse anti$\beta$-actin (1:5000, Sigma-Aldrich).
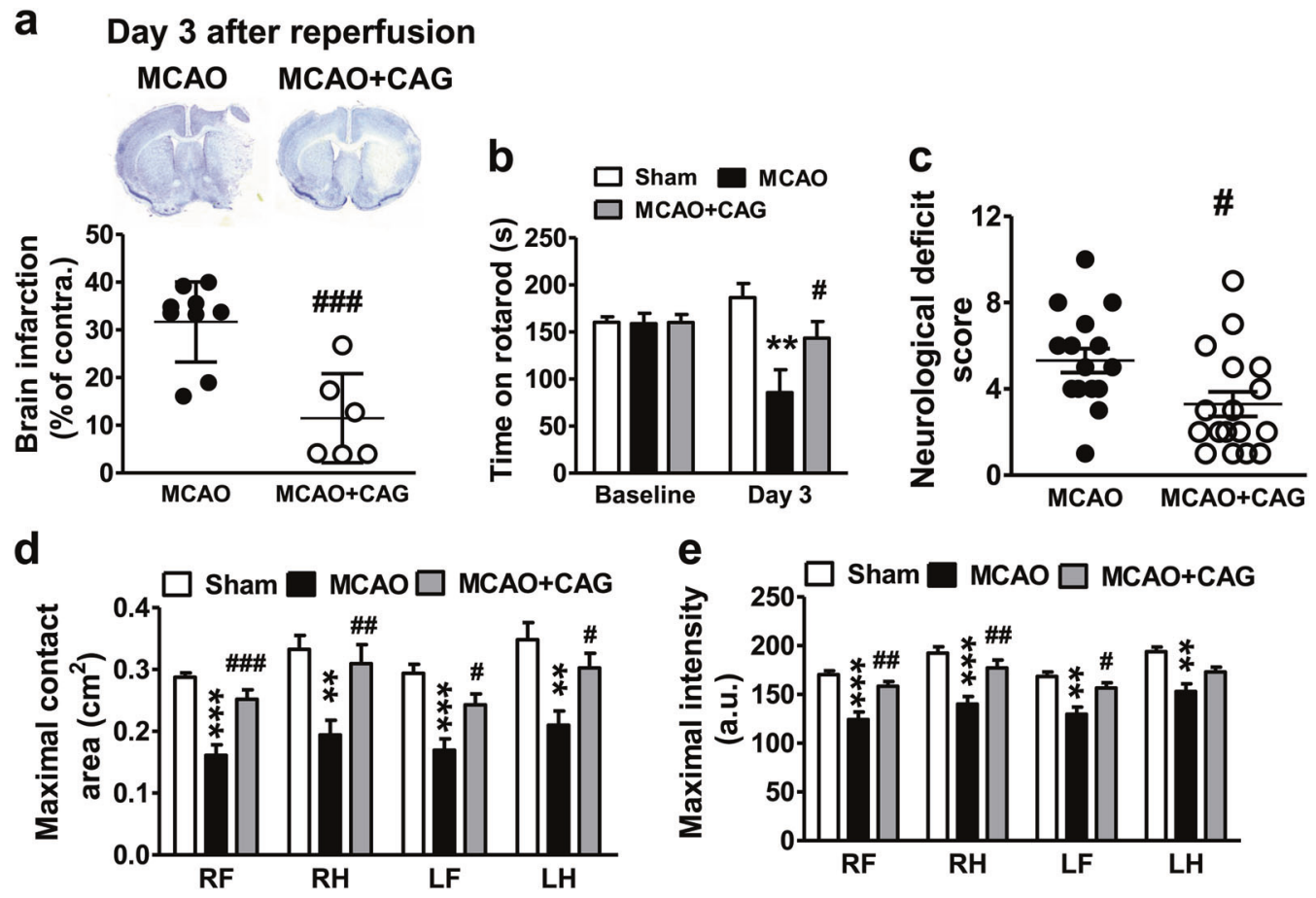

f
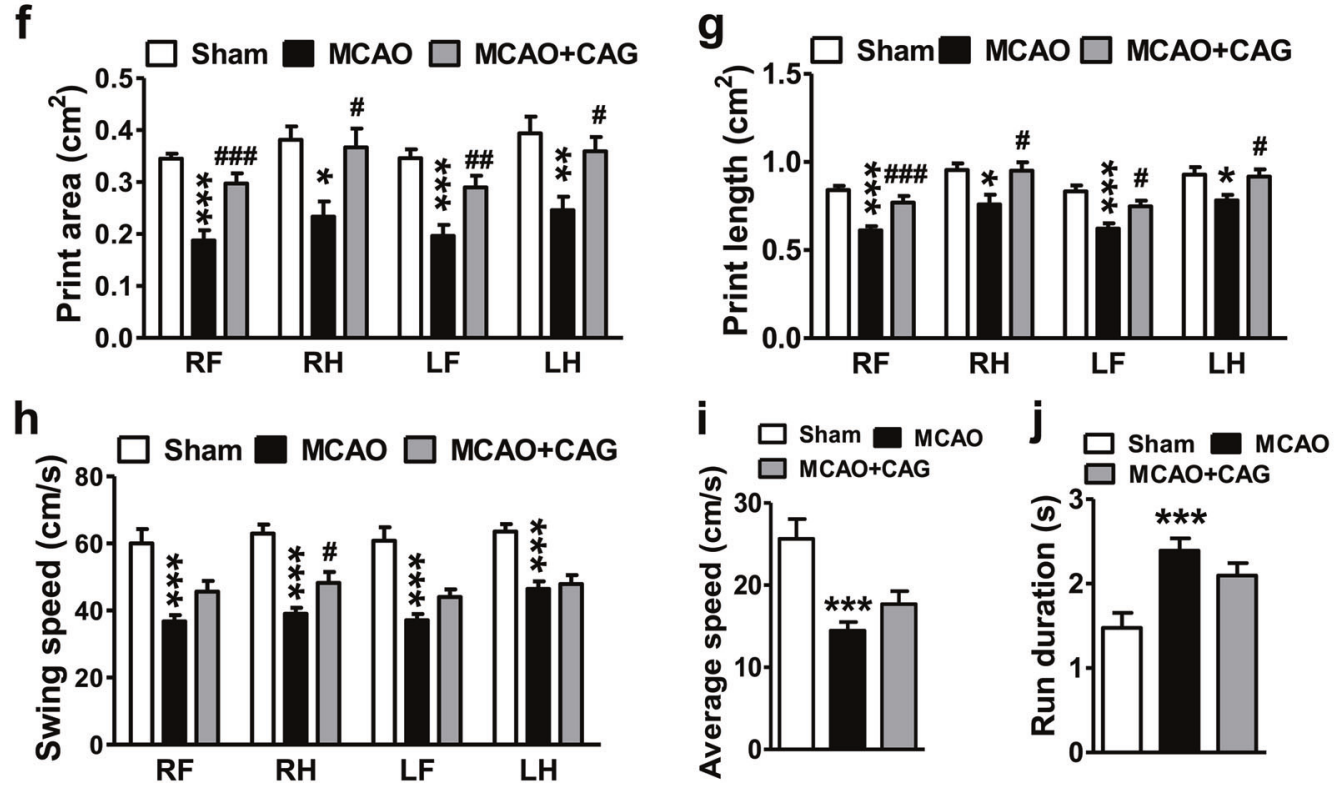

Fig. 2 CAG $(20 \mathrm{mg} / \mathrm{kg})$ reduced brain infarction and ameliorated functional deficits in MCAO mice on Day 3 after reperfusion. a CAG robustly reduced brain infarction, as detected by Nissl staining. CAG prolonged the time spent on the rotarod (b), reduced neurological deficit scores (c) and ameliorated deficits in gait parameters (d-h) in MCAO mice. $\mathbf{i}, \mathbf{j}$ CAG did not affect the average speed or run duration of MCAO mice. RF, right forepaw; $\mathrm{RH}$, right hind paw; $\mathrm{LF}$, left forepaw; $\mathrm{LH}$, left hind paw. ${ }^{*} P<0.05$; ${ }^{* *} P<0.01$; ${ }^{* *} P<0.001$ compared with the sham control group; ${ }^{*} P<$

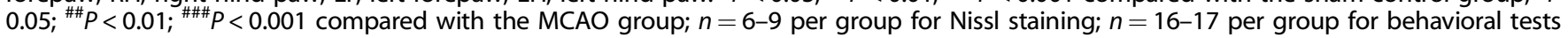




\section{SIRT1 activity assay}

A fluorescence-based assay was used to evaluate the effect of CAG on SIRT1 activity [18]. CAG and resveratrol were dissolved in DMSO to generate a $10 \mathrm{mM}$ stock solution. The assay was run in black 96-well plates in a final reaction volume of $100 \mu \mathrm{L}$. Reactions were performed in assay buffer $(25 \mathrm{mM}$ Tris, $\mathrm{pH} 8.0,137 \mathrm{mM} \mathrm{NaCl}, 2.7 \mathrm{mM} \mathrm{KCl}$ and 1 $\mathrm{mM} \mathrm{MgCl} 2$ ) with $1 \mu \mathrm{M}$ SIRT1, $50 \mu \mathrm{M}$ RHKK-AMC and $750 \mu \mathrm{M} \mathrm{NAD}^{+}$at $25^{\circ} \mathrm{C}$ for $45 \mathrm{~min}$ and were stopped with stop/developing solution $(50 \mu \mathrm{L})$ containing $0.5 \mu \mathrm{g} / \mu \mathrm{L}$ trypsin and $10 \mathrm{mM}$ nicotinamide. After incubation for $30 \mathrm{~min}$, the fluorescence was analyzed using a microplate reader (POLARstar OPTIMA, BMG Labtech, Ortenberg, Germany) with excitation at $340 \mathrm{~nm}$ and emission at $490 \mathrm{~nm}$.

\section{Immunoprecipitation}

Lysates from the ipsilateral hemisphere were immunoprecipitated overnight at $4{ }^{\circ} \mathrm{C}$ with mouse anti-p65 (1:100, Cell Signaling Technology)/normal IgG (1:100, Sigma-Aldrich), mouse anti-p53 (1:50, Santa Cruz Biotechnology)/normal IgG (1:50, Sigma-Aldrich), and then conjugated to nProtein A Sepharose ${ }^{\mathrm{TM}} 4$ Fast Flow/Protein $G$ Sepharose ${ }^{T M} 4$ Fast Flow (GE Healthcare Bio-Science AB, Uppsala, Sweden) for $2 \mathrm{~h}$ at $4{ }^{\circ} \mathrm{C}$. The complexes were washed three times with lysis buffer, denatured and subjected to Western blotting using rabbit anti-acetyl-lysine (1:1000, Cell Signaling Technology), mouse anti-p53 (1:500, Santa Cruz Biotechnology), rabbit anti-NF-KB p65 (acetyl K310) (1:3000, Abcam), and mouse anti-p65 (1:1000, Cell Signaling Technology).

Statistical analysis

The data are expressed as the mean \pm SEM. Statistical analysis was performed using GraphPad Prism 7.0. Rotarod performance was analyzed using two-way repeated-measures analysis of variance. Comparisons between two groups and multiple groups were evaluated by Student's $t$ test and one-way analysis of variance followed by Tukey's post hoc comparisons, respectively. Differences were considered statistically significant at $P<0.05$. a
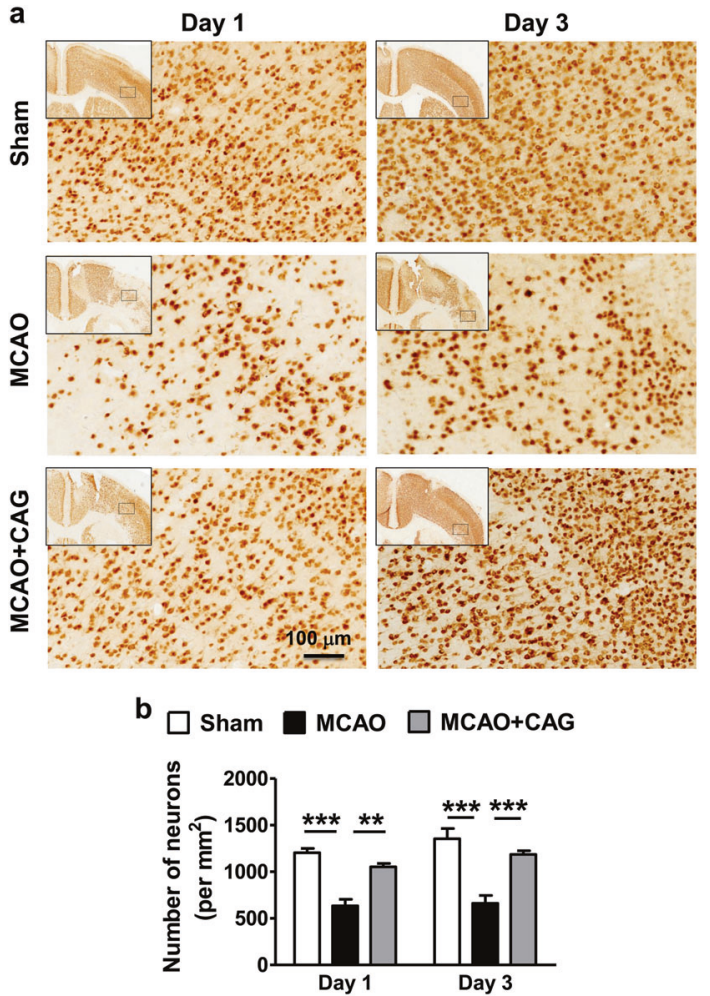

Fig. 3 CAG ameliorated neuronal cell loss in the ischemic cortex on Days 1 and 3 after reperfusion. a NeuN-positive neurons were detected by immunohistochemistry. Representative images showing that CAG prevented neuronal cell loss in the ischemic cortex on Days 1 and Day 3 after reperfusion. b Quantitative results. ${ }^{* *} P<0.01$; ***P $<0.001 ; n=6$ per group a
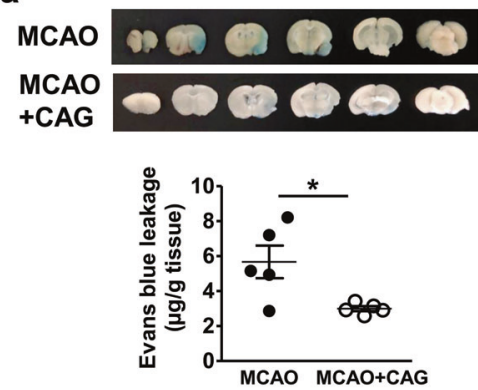

C

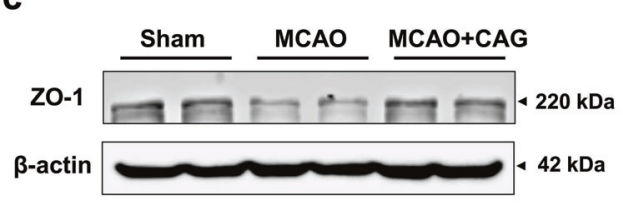

d

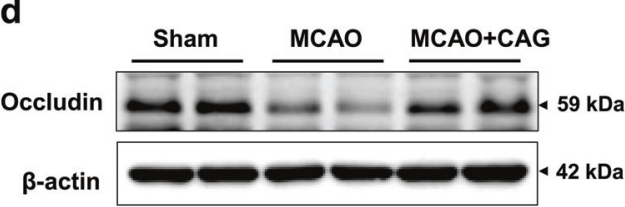

b
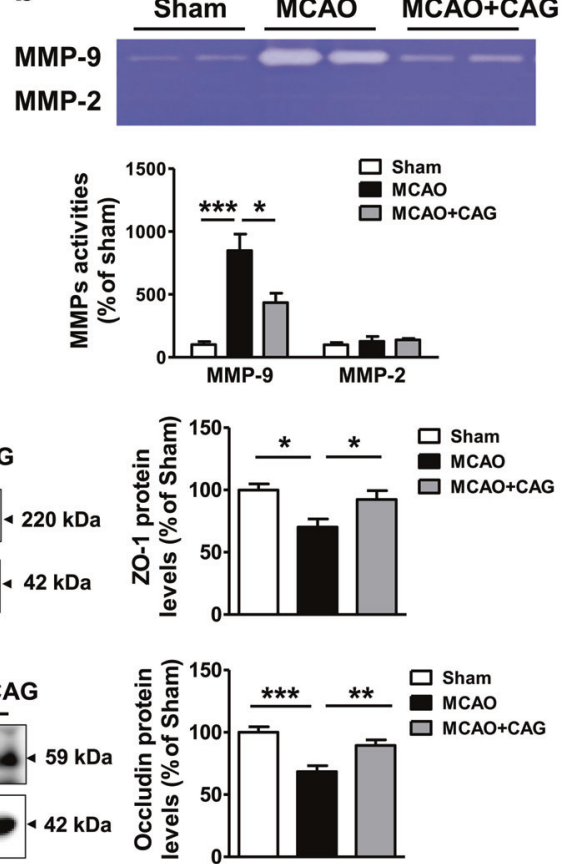

Fig. 4 CAG attenuated MCAO-induced BBB disruption, MMP-9 elevation, and tight junction protein degradation on Day 1 after reperfusion. a CAG attenuated MCAO-induced Evans blue extravasation in the ischemic hemisphere. $\mathbf{b}$ Treatment with CAG inhibited the elevation of MMP-9 activity, as assessed by gelatin zymography. Western blotting showed that CAG restored the protein levels of ZO-1 (c) and Occludin (d) downregulated by MCAO. ${ }^{*} P<0.05 ;{ }^{*} P<0.01 ;{ }^{* * *} P<0.001 ; n=5-9$ per group 
a

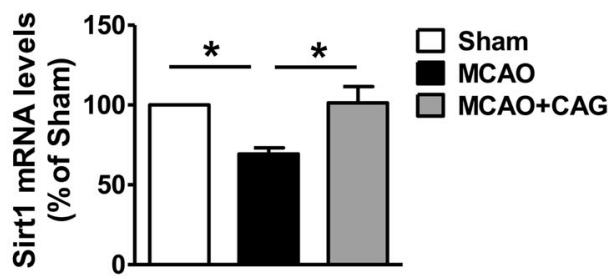

b

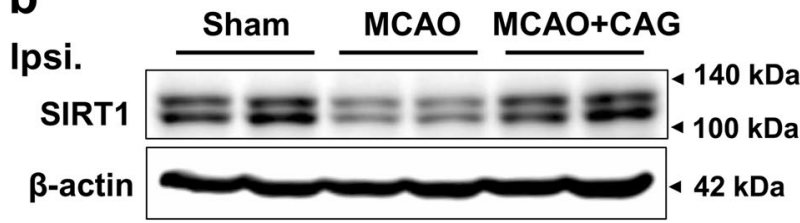

Contra.
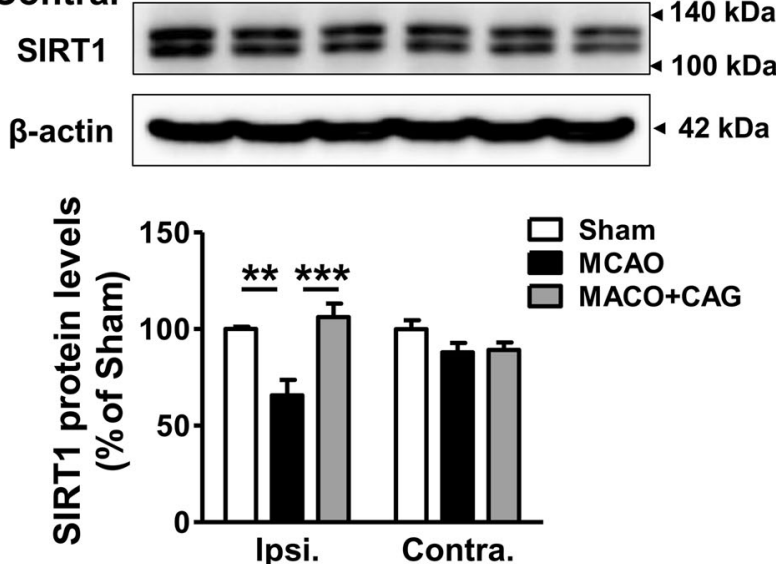

C

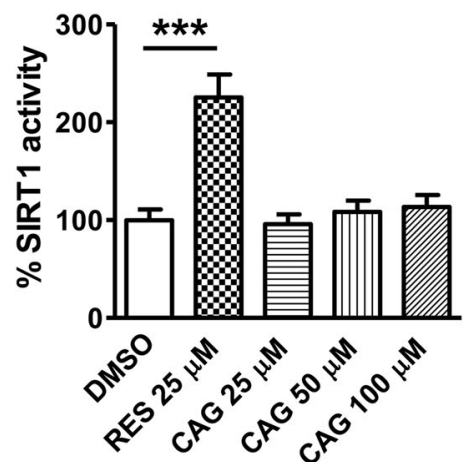

Fig. 5 CAG increased SIRT1 expression in the ischemic brain on Day 1 after reperfusion. CAG completely prevented the MCAO-induced downregulation of SIRT1 mRNA (a) and protein expression (b) in the ischemic brain, as measured by real-time PCR and Western blotting, respectively. SIRT1 protein levels remained unchanged in the contralateral hemisphere of MCAO mice treated with or without CAG. c SIRT1 activity was detected by a fluorescence-based assay. Compared with resveratrol (RES), which was used as a positive control, CAG did not directly activate SIRT1 activity. ${ }^{*} P<0.05 ;{ }^{*} P<$ $0.01 ;{ }^{* * *} P<0.001 ; n=12-14$ per group for $\mathrm{a}$ and $\mathrm{b} ; n=4$ for $\mathrm{c}$

\section{RESULTS}

CAG ameliorated functional deficits, reduced brain infarction and attenuated neuronal loss in MCAO mice

We first evaluated the protective effects of $5 \mathrm{mg} / \mathrm{kg}, 10 \mathrm{mg} / \mathrm{kg}$ and $20 \mathrm{mg} / \mathrm{kg}$ CAG on Day 1 after reperfusion. MCAO induced a dramatic decrease in the time that mice were able to stay on the accelerating rotarod, and $5 \mathrm{mg} / \mathrm{kg}$ and $20 \mathrm{mg} / \mathrm{kg}$ CAG significantly

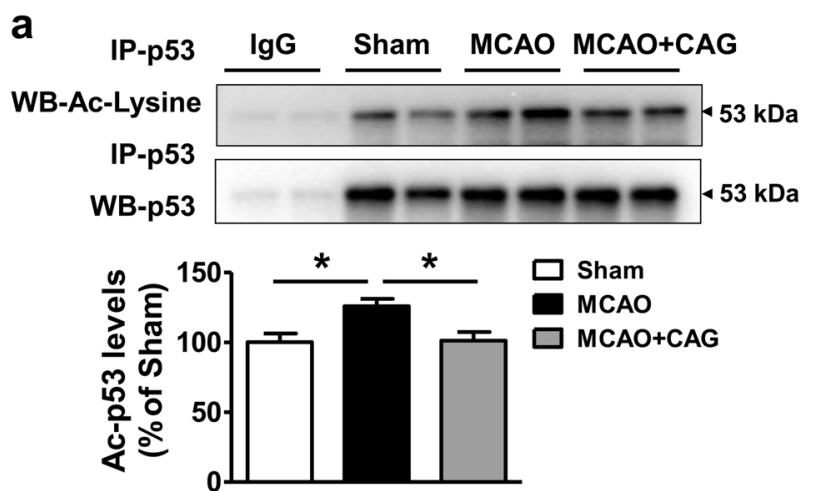

b

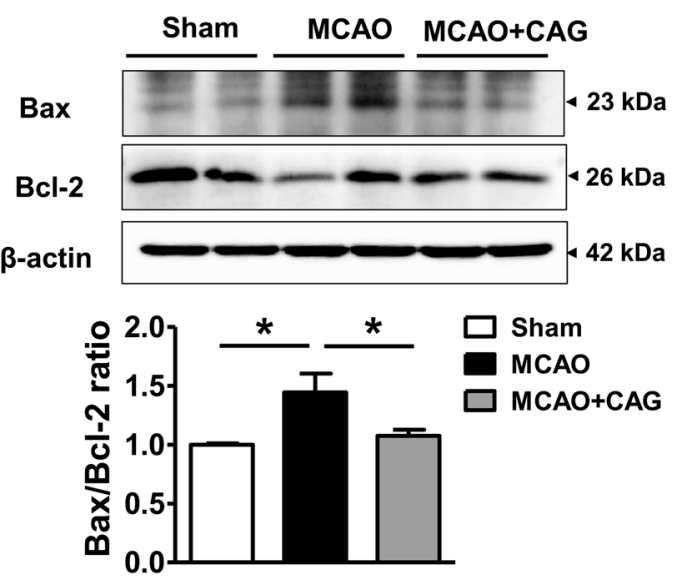

Fig. 6 CAG suppressed MCAO-mediated induction of the p53 apoptotic signaling pathway on Day 1 after reperfusion. a Immunoprecipitation was performed to pool p53 from brain lysates, and then Western blotting was used to measure the acetylation of p53. Acetylated p53 was increased in the ischemic brain, and this increase was suppressed by CAG treatment. b The ratio of $\mathrm{Bax}$ to $\mathrm{BCl}-2$ was markedly increased in the ischemic cortex, and CAG treatment robustly reduced the $\mathrm{Bax} / \mathrm{Bcl}-2$ ratio. ${ }^{*} P<0.05, n=6-8$ per group

prolonged the time spent on the rotarod (from $29.6 \mathrm{~s}$ to $75.0 \mathrm{~s}$ and 88.0 s, respectively; Fig. 1b). CAG also markedly reduced neurological deficit scores in a dose-dependent manner (Fig. 1c). In addition, $20 \mathrm{mg} / \mathrm{kg}$ CAG robustly reduced the brain infarct volume from $52.6 \mathrm{~mm}^{3}$ to $13.7 \mathrm{~mm}^{3}$ (Fig. $1 \mathrm{~d}$, e). These results suggested that $20 \mathrm{mg} / \mathrm{kg}$ CAG provided the strongest beneficial effects against brain ischemic injury. Therefore, $20 \mathrm{mg} / \mathrm{kg}$ CAG was used in subsequent studies.

Next, we assessed whether $20 \mathrm{mg} / \mathrm{kg}$ CAG was neuroprotective on Day 3 after reperfusion. As expected, CAG markedly reduced brain infarction, improved the rotarod performance and decreased the neurological deficit scores of MCAO mice (Fig. 2a-c). Furthermore, CAG significantly ameliorated gait impairment in MCAO mice. MCAO markedly decreased the maximal contact area, maximal intensity, print area, print length and swing speed of the mice (Fig. 2d-h). CAG treatment significantly increased the maximal contact area, print area and print length (of all four paws), maximal intensity (of the right paws and left front paw), and swing speed (of the right hind paw) of MCAO mice. In addition, MCAO decreased the average speed and prolonged the run duration of the mice. However, CAG treatment did not affect these two parameters (Fig. 2i, j).

As detected by NeuN staining, MCAO induced a severe loss of neuronal cells in the ischemic cortex on Days 1 and 3 after reperfusion, and neuronal cell loss was robustly ameliorated by CAG treatment (Fig. 3). 


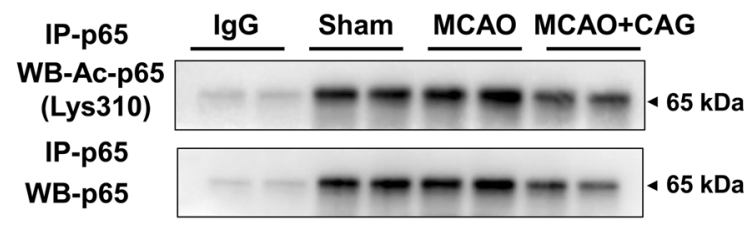

C

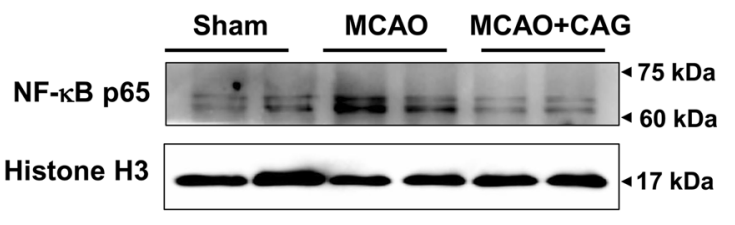

e TNF- $\alpha$ mRNA

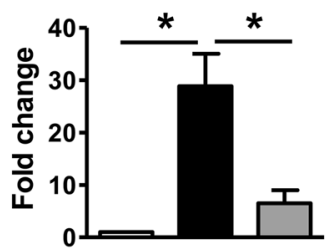

b

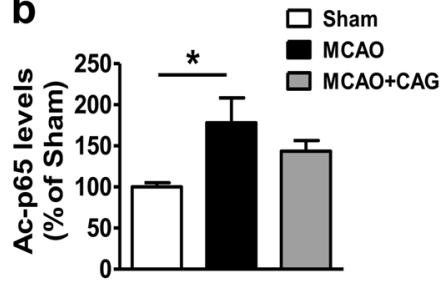

d
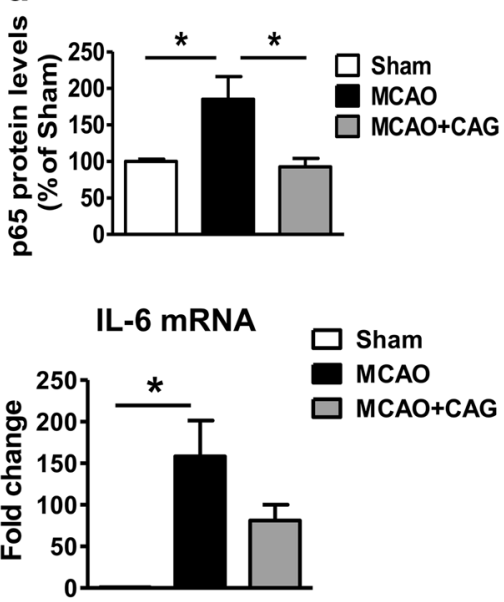

Fig. 7 CAG suppressed MCAO-induced NF-кB activation and neuroinflammation on Day 1 after reperfusion. $\mathbf{a}$, $\mathbf{b}$ Immunoprecipitation was performed to pool p65 from brain lysates, and then Western blotting was used to measure the acetylation of p65 at Lys310. The levels of acetylated p65 at Lys310 were increased in the ischemic brain, and this increase was partially reduced by CAG treatment. c, $\mathbf{d}$ Western blotting was used to detect p65 in nuclear extracts. CAG completely inhibited MCAO-induced p65 nuclear translocation. e Real-time PCR showed that the mRNA levels of TNF- $\alpha$ and IL-1 $\beta$ were significantly increased in the ischemic brain and markedly suppressed by CAG treatment. CAG did not affect IL- 6 mRNA levels in the brains of MCAO mice. ${ }^{*} P<0.05, n=6-8$ per group

CAG reduced MMP-9 activity, prevented tight junction protein degradation and attenuated BBB disruption

Evans blue extravasation is commonly used to evaluate the permeability of the BBB. An obvious increase in Evans blue content was noted in the ipsilateral hemisphere of MCAO mice on Day 1 after reperfusion (Fig. 4a). CAG treatment robustly reduced Evans blue content in the ischemic brain from 5.7 to $3.0 \mu \mathrm{g} / \mathrm{g}$. Severe BBB disruption has been linked to the upregulation of MMP-9 in the ischemic brain. As detected by gelatin zymography, MMP-9 activity was dramatically upregulated in the ischemic cortex of MCAO mice, and CAG markedly suppressed the upregulation of MMP-9 activity (Fig. 4b). MMP-2 activity remained unaffected by MCAO or CAG treatment. Tight junction proteins, the key components of the BBB structure, are substrates of MMP-9. Concomitant with the upregulation of MMP-9 activity, protein levels of tight junction ZO-1 and Occludin were markedly reduced in the ischemic brain, and CAG nearly completely prevented tight junction protein degradation (Fig. 4c, d).

CAG upregulated SIRT1 expression in the ischemic brain but did not directly activate SIRT1

Compared with those in the sham group, both the mRNA and protein levels of SIRT1 were markedly decreased in the ischemic brain on Day 1 after reperfusion (Fig. 5a, b). CAG treatment completely prevented the downregulation of SIRT1 mRNA and protein expression after MCAO. However, CAG did not directly affect the enzymatic activity of SIRT1, as assessed by an in vitro SIRT1 activity assay with resveratrol, which was used as a positive control (Fig. 5c). SIRT1 protein levels remained unchanged in the contralateral hemisphere of MCAO mice with or without CAG treatment (Fig. 5b).
CAG reduced p53 acetylation and suppressed p53-mediated apoptotic signaling pathway in the ischemic brain

SIRT1 can deacetylate p53 and inhibit its transcriptional activity. Concomitant with the downregulation of SIRT1 expression, MCAO significantly increased the acetylation of p53 in the ischemic brain on Day 1 after reperfusion (Fig. 6a). As a result, the ratio of proapoptotic Bax to anti-apoptotic Bcl-2 was increased after MCAO (Fig. 6b). As anticipated, CAG treatment reduced the acetylation level of p53 and subsequently decreased the ratio of $\mathrm{Bax} / \mathrm{Bcl}-2$ in the ischemic brain.

CAG suppressed NF-KB activation and inhibited neuroinflammation in the ischemic brain

NF-KB p65 is a substrate of SIRT1. On Day 1 after reperfusion, MCAO markedly increased the acetylation of p65 at Lys310 in the ischemic brain, and this upregulation was suppressed by CAG, although the effect of CAG was not statistically significant (Fig. 7a, b). Concurrently, CAG completely suppressed MCAO-induced p65 nuclear translocation in the ischemic brain (Fig. 7c, d). As a result, CAG robustly inhibited MCAO-induced neuroinflammation by reducing TNF- $\alpha$ and IL-1 $\beta$ mRNA levels on Day 1 (Fig. 7e) and suppressing the activation of microglia and astrocytes in the ischemic brain on Day 3 after reperfusion (Fig. 8). CAG did not affect IL- 6 mRNA in the ischemic brain on Day 1.

\section{DISCUSSION}

This study demonstrated that CAG exerted potent neuroprotective effects against ischemic brain injury in a mouse model of transient focal cerebral ischemia. CAG reduced the brain infarct volume, prevented neuronal cell loss, attenuated BBB damage, and improved 
a
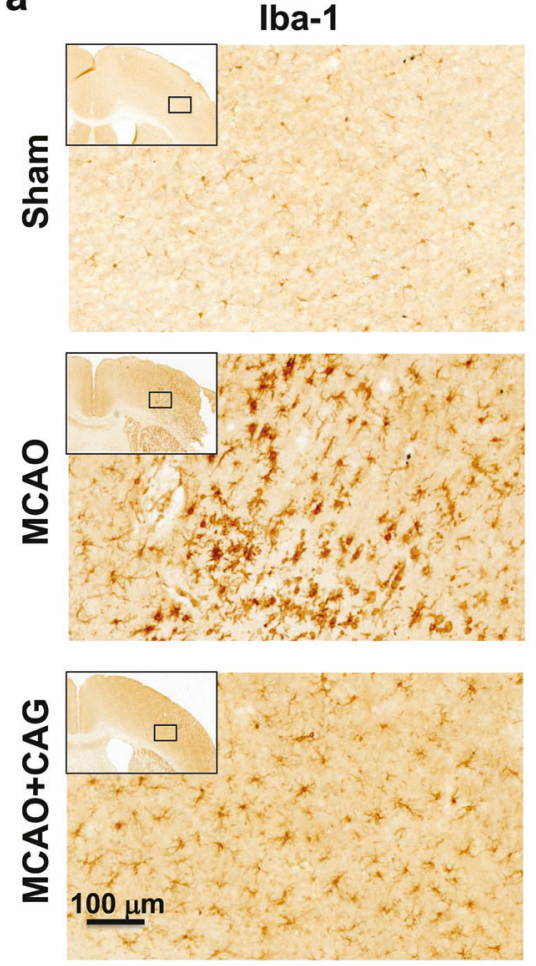

Iba-1
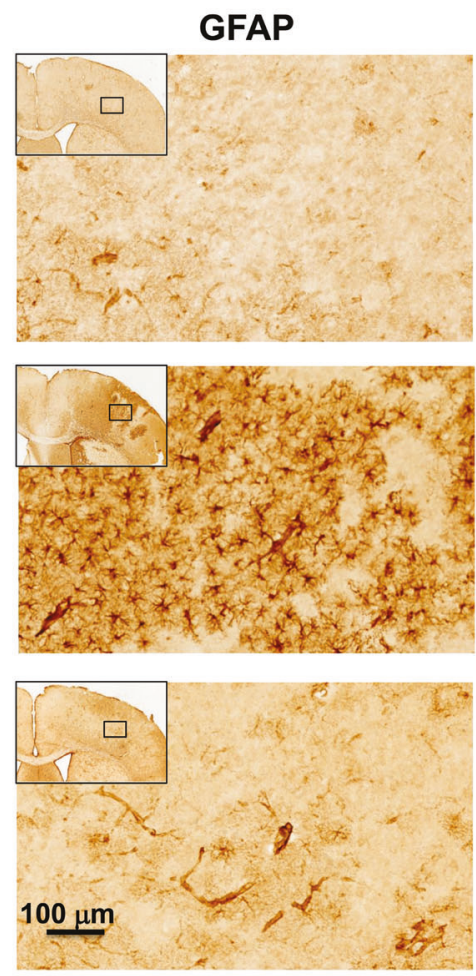

b
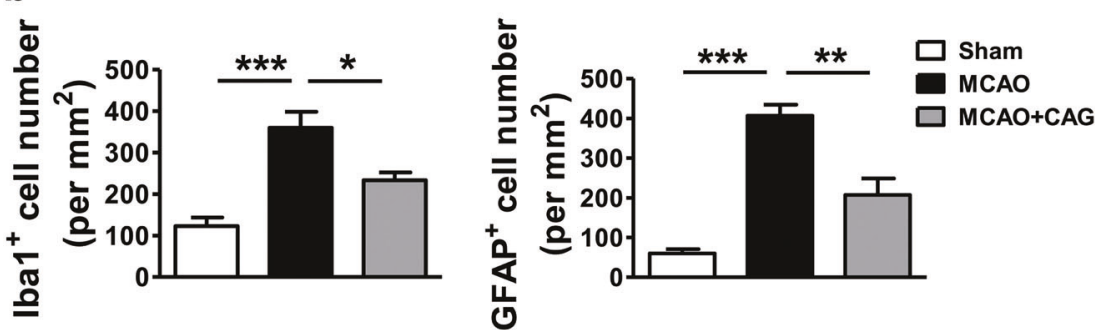

Fig. 8 CAG inhibited the activation of microglial cells and astrocytes in the ischemic brain on Day 3 after reperfusion. a As assessed by immunohistochemical staining, MCAO induced the activation of microglial cells $\left(\mathrm{Iba}^{+}\right)$and astrocytes $\left(\mathrm{GFAP}^{+}\right)$in the ischemic cortex compared with that in the sham control group, and the activation of these cells was markedly inhibited by CAG treatment. $\mathbf{b}$ Quantitative results. ${ }^{*} P<0.05 ;{ }^{* *} P<0.01 ;{ }^{* *} P<0.001 ; n=6$ per group

functional deficits in MCAO mice. These beneficial effects of CAG lasted for at least 3 days after reperfusion. CAG upregulated SIRT1 expression, reduced the acetylation of p53, suppressed NF-kB activation, and inhibited apoptosis and neuroinflammation in the ischemic brain. These effects may contribute to the overall beneficial effects of CAG against brain ischemia.

SIRT1 is an NAD ${ }^{+}$-dependent class III histone deacetylase and regulates various biological processes, including oxidative stress, apoptosis, inflammation, energy metabolism, autophagy, and mitochondrial function [19]. SIRT1 is abundantly expressed in the brain and plays an important role in neuroprotection under a variety of neurological conditions, including stroke [20]. Previous studies have shown that pharmacological and genetic SIRT1 manipulations alter the outcome of stroke in rodent models [7-9]. In our paradigm, MCAO triggered a significant reduction in both the mRNA and protein levels of SIRT1 in the ischemic brain, whereas CAG treatment completely restored the mRNA and protein expression of SIRT1. In addition, CAG significantly improved functional outcomes and reduced brain infarction in MCAO mice. Therefore, the mechanism underlying the beneficial effects of CAG against cerebral ischemia likely involves SIRT1 upregulation.
In addition to histones, SIRT1 targets nonhistone protein substrates to modulate a variety of biological processes. SIRT1 can deacetylate p53 and NF-kB p65, which are key factors that mediate apoptotic and inflammatory pathways, respectively $[21,22]$. SIRT1 deacetylates p53 at multiple lysine residues and reduces its transcriptional activity [23]. SIRT1 can also inhibit NF-KB activation through the deacetylation of p65 at Lys310 [24]. The acetylation of p53 and p 65 has also been observed under ischemic conditions $[25,26]$. Furthermore, it has been shown that SIRT1 protects the brain against cerebral ischemic damage through deacetylation and the subsequent inhibition of p53-induced apoptosis and p65-mediated neuroinflammation [6]. In agreement with these findings, our results showed a significant increase in p53 and p65 acetylation in the ischemic brain. Concurrent with SIRT1 upregulation, CAG treatment induced p53 deacetylation after MCAO. As a result, CAG inhibited post-ischemic p53dependent apoptosis by reducing the ratio of pro-apoptotic Bax to anti-apoptotic Bcl-2 in the ischemic brain. Although CAG did not significantly affect the acetylation of p65 at Lys310 in the ischemic brain, CAG suppressed NF-KB activation and subsequently reduced the expression of pro-inflammatory factors and the activation of glial cells after MCAO. Therefore, other 
pharmacological mechanisms may be involved in the antiinflammatory effects of CAG.

Interestingly, we found that CAG may not be a direct SIRT1 activator because it did not increase SIRT1 enzyme activity compared with resveratrol in an in vitro assay. Therefore, instead of directly activating the enzyme, CAG may enhance SIRT1 activity by upregulating its expression in the ischemic brain. It should also be noted that increases in SIRT1 expression may not always lead to increases in its enzymatic activity. A previous study demonstrated that SIRT1-mediated neuroprotection can be independent of its deacetylase activity and suggested that the enzymatic activity of SIRT1 may only be partially responsible for its protective effects [27].

Stroke induces BBB disruption, which has been linked to increased MMP-9 activity and subsequent degradation of tight junction proteins and basal components of the BBB [28]. SIRT1 has been reported to play a critical role in BBB permeability. Overexpressing Sirt1 or increasing SIRT1 activity has been shown to stabilize tight junction proteins and reduce BBB leakage in aging mice [29]. SIRT1 inhibition exacerbates BBB disruption by increasing endothelial cell apoptosis and enhancing MMP-9 gelatinase activity after subarachnoid hemorrhage in rats [30]. Calcium/calmodulin-dependent protein kinase kinase (CaMKK) can activate SIRT1, and the inhibition of CaMKK concurrently suppresses SIRT1 activation. A recent study showed that CaMKK inhibition can reduce endothelial cell viability and aggravate BBB disruption after cerebral ischemia [31]. In the present study, concomitant with the upregulation of SIRT1 expression, CAG suppressed MCAO-induced MMP-9 activity and the degradation of the tight junction proteins ZO-1 and Occludin. As a result, CAG attenuated BBB breakdown in MCAO mice.

This study has demonstrated that CAG can ameliorate postischemic brain injury and functional deficits in mice after cerebral ischemia. The overall beneficial effects of CAG against cerebral ischemia most likely involve the upregulation of SIRT1 expression, the deacetylation of $\mathrm{p53}$, the suppression of NF-KB activation, and the subsequent inhibition of apoptosis and neuroinflammation. Together, these findings suggest that upregulating or activating SIRT1 may be a promising therapeutic strategy for improving stroke outcomes, and clinical trials of CAG for this brain disorder are warranted.

\section{ACKNOWLEDGEMENTS}

This study was supported by the National Natural Science Foundation of China (81873029).

\section{AUTHOR CONTRIBUTIONS}

$M L$ and ZFW designed the study. ML, SCL, BKD, YXZ and $\mathrm{HZH}$ performed the experiments. $\mathrm{ML}, \mathrm{SCL}, \mathrm{DXL}, \mathrm{ZJK}$ and $\mathrm{ZFW}$ wrote the manuscript.

\section{ADDITIONAL INFORMATION}

Competing interests: The authors declare no competing interests.

\section{REFERENCES}

1. Wang W, Jiang B, Sun $H$, Ru X, Sun D, Wang $L$, et al. Prevalence, incidence, and mortality of stroke in China: results from a nationwide population-based survey of 480687 adults. Circulation. 2017;135:759-71.

2. Bordone L, Guarente L. Calorie restriction, SIRT1 and metabolism: understanding longevity. Nat Rev Mol Cell Biol. 2005;6:298-305.

3. Zhang F, Wang S, Gan L, Vosler PS, Gao Y, Zigmond MJ, et al. Protective effects and mechanisms of sirtuins in the nervous system. Prog Neurobiol. 2011;95:373-95.

4. Koronowski KB, Perez-Pinzon MA. Sirt1 in cerebral ischemia. Brain Circ. 2015;1:69-78.

5. Zhang JF, Zhang YL, Wu YC. The role of Sirt1 in ischemic stroke: pathogenesis and therapeutic strategies. Front Neurosci. 2018;12:833.
6. Hernandez-Jimenez M, Hurtado O, Cuartero MI, Ballesteros I, Moraga A, Pradillo $J M$, et al. Silent information regulator 1 protects the brain against cerebral ischemic damage. Stroke. 2013;44:2333-7.

7. Hattori $Y$, Okamoto $Y$, Nagatsuka $K$, Takahashi R, Kalaria RN, Kinoshita $M$, et al. SIRT1 attenuates severe ischemic damage by preserving cerebral blood flow. Neuroreport. 2015;26:113-7.

8. Raval AP, Dave KR, Perez-Pinzon MA. Resveratrol mimics ischemic preconditioning in the brain. J Cereb Blood Flow Metab. 2006;26:1141-7.

9. Della-Morte D, Dave KR, DeFazio RA, Bao YC, Raval AP, Perez-Pinzon MA. Resveratrol pretreatment protects rat brain from cerebral ischemic damage via a sirtuin 1-uncoupling protein 2 pathway. Neuroscience. 2009;159:993-1002.

10. Ren S, Zhang H, Mu Y, Sun M, Liu P. Pharmacological effects of Astragaloside IV: a literature review. J Tradit Chin Med. 2013;33:413-6.

11. Zhou RN, Song YL, Ruan JQ, Wang YT, Yan R. Pharmacokinetic evidence on the contribution of intestinal bacterial conversion to beneficial effects of astragaloside IV, a marker compound of astragali radix, in traditional oral use of the herb. Drug Metab Pharmacokinet. 2012;27:586-97.

12. Zhao Y, Li Q, Zhao W, Li J, Sun Y, Liu K, et al. Astragaloside IV and cycloastragenol are equally effective in inhibition of endoplasmic reticulum stress-associated TXNIP/NLRP3 inflammasome activation in the endothelium. J Ethnopharmacol. 2015;169:210-8.

13. Ma PK, Wei BH, Cao YL, Miao Q, Chen N, Guo CE, et al. Pharmacokinetics, metabolism, and excretion of cycloastragenol, a potent telomerase activator in rats. Xenobiotica. 2017;47:526-37.

14. Gu M, Zhang S, Zhao Y, Huang J, Wang Y, Li Y, et al. Cycloastragenol improves hepatic steatosis by activating farnesoid $X$ receptor signalling. Pharmacol Res. 2017;121:22-32.

15. Wang Z, Tsai LK, Munasinghe J, Leng Y, Fessler EB, Chibane F, et al. Chronic valproate treatment enhances postischemic angiogenesis and promotes functional recovery in a rat model of ischemic stroke. Stroke. 2012;43:2430-6.

16. Dou B, Zhou W, Li S, Wang L, Wu X, Li Y, et al. Buyang Huanwu decoction attenuates infiltration of natural killer cells and protects against ischemic brain injury. Cell Physiol Biochem. 2018;50:1286-300.

17. Wang Z, Leng Y, Tsai LK, Leeds $P$, Chuang DM. Valproic acid attenuates bloodbrain barrier disruption in a rat model of transient focal cerebral ischemia: the roles of HDAC and MMP-9 inhibition. J Cereb Blood Flow Metab. 2011;31:52-7.

18. Wu J, Zhang D, Chen L, Li J, Wang J, Ning C, et al. Discovery and mechanism study of SIRT1 activators that promote the deacetylation of fluorophore-labeled substrate. J Med Chem. 2013;56:761-80.

19. Horio Y, Hayashi T, Kuno A, Kunimoto R. Cellular and molecular effects of sirtuins in health and disease. Clin Sci (Lond). 2011;121:191-203.

20. Ramadori G, Lee CE, Bookout AL, Lee S, Williams KW, Anderson J, et al. Brain SIRT1: anatomical distribution and regulation by energy availability. J Neurosci. 2008;28:9989-96.

21. Sirotkin AV, Dekanova $P$, Harrath $A H$, Alwasel $S H$, Vasicek D. Interrelationships between sirtuin 1 and transcription factors p53 and NF-kappaB (p50/p65) in the control of ovarian cell apoptosis and proliferation. Cell Tissue Res. 2014;358:627-32.

22. Liu TF, McCall CE. Deacetylation by SIRT1 Reprograms inflammation and cancer. Genes Cancer. 2013;4:135-47.

23. Vaziri H, Dessain SK, Ng Eaton E, Imai SI, Frye RA, Pandita TK, et al. hSIR2(SIRT1) functions as an NAD-dependent p53 deacetylase. Cell. 2001;107:149-59.

24. Yeung F, Hoberg JE, Ramsey CS, Keller MD, Jones DR, Frye RA, et al. Modulation of NF-kappaB-dependent transcription and cell survival by the SIRT1 deacetylase. EMBO J. 2004;23:2369-80.

25. Raz L, Zhang QG, Han D, Dong Y, De Sevilla L, Brann DW. Acetylation of the proapoptotic factor, p53 in the hippocampus following cerebral ischemia and modulation by estrogen. PLoS One. 2011;6:e27039.

26. Lanzillotta A, Sarnico I, Ingrassia R, Boroni F, Branca C, Benarese M, et al. The acetylation of RelA in Lys310 dictates the NF-kappaB-dependent response in post-ischemic injury. Cell Death Dis. 2010;1:e96.

27. Pfister JA, Ma C, Morrison BE, D'Mello SR. Opposing effects of sirtuins on neuronal survival: SIRT1-mediated neuroprotection is independent of its deacetylase activity. PLoS One. 2008;3:e4090.

28. Chaturvedi M, Kaczmarek L. Mmp-9 inhibition: a therapeutic strategy in ischemic stroke. Mol Neurobiol. 2014;49:563-73.

29. Stamatovic SM, Martinez-Revollar G, Hu A, Choi J, Keep RF, Andjelkovic AV. Decline in Sirtuin-1 expression and activity plays a critical role in blood-brain barrier permeability in aging. Neurobiol Dis. 2019;126:105-16.

30. Zhou XM, Zhang X, Zhang XS, Zhuang Z, Li W, Sun Q, et al. SIRT1 inhibition by sirtinol aggravates brain edema after experimental subarachnoid hemorrhage. J Neurosci Res. 2014;92:714-22.

31. Sun P, Bu F, Min JW, Munshi Y, Howe MD, Liu L, et al. Inhibition of calcium/ calmodulin-dependent protein kinase kinase (CaMKK) exacerbates impairment of endothelial cell and blood-brain barrier after stroke. Eur J Neurosci. 2019;49:27-39. 